\title{
Research on Stiffness of the Hull Beam Model Based on ADAMS
}

\author{
Yanshi He, Fangzhen Song and Haiyan Shao \\ School of Mechanical Engineering, University of Jinan, Jinan, 250022, China
}

\begin{abstract}
A piecewise rigid body model of the ship is proposed, which makes the ship, a elastic body, can be analyzed in dynamics software like ADAMS and the analysis efficiency is improved. The equivalent stiffness in the model is more close to the actual situation, so it improves the precision of calculating natural frequency in the vibration characteristic analysis. Having been used in ADAMS, the modelling method turns out to be more accurate processing method than common hull beam section unit method. The processing method be used for the dynamics analysis of the similar structure.
\end{abstract}

\section{Introduction}

Due to the length of the ship is much larger than the high and wide of the ship, so the ship is often simplified as beam by scholar to the study of the ship vibration problem . The formula, characteristics and law of the vibration of the beam can be applied to the study of hull vibration, when the beam model is used to deal with the vibration problems of the hull [1], [2], [3]. It makes the research easier and improves the efficiency of the study.

However, the hull structure is quite complex, so it can not be fully equivalent to the beam. Therefore, when the hull simplify to hull beam model, we should first to consider that how to get a more accurate model to simulate the actual quality and stiffness of the hull. For quality, the influence of different internal hull structure is not obvious, and the change of the internal structure does not change the external hull shape. It is not difficult to get the rule of the influences of the entrained water [4], [5]. So this paper focus on the stiffness simplified method of the hull beam model. In order to facilitate the calculation, this paper uses the multi rigid body dynamics software ADAMS to assist the completion of the study [6].

For the the vibration analysis of chain structure such as hull beam, there have concentrated mass method, transfer matrix method, finite element method and so on $[7,8,9]$. For the method of simplified model, the literature $[10,11]$ by using the finite element method to achieve the equivalent model of the mechanism .The transfer matrix of rigid body system connected by spring and hinge is given in literature [12].

No matter what kind of simplified method, the treatment of equivalent stiffness is an important part of the study. The accuracy of the equivalent stiffness directly affects the results of the entire study whether is correct and whether the conclusion of the research makes sense.

\section{The common model of hull beam}

The hull beam model for calculating the total vibration, which is a row of hull beam elements connected by nodes [13].

For each simplified beam element, the selection rules of the raw data as follows:

(1) Each simplified beam element is considered as a uniform straight beam;

(2) The arithmetic mean of the moment of inertia of the section at both ends of the beam is taken as the moment of inertia of each beam section;

(3) The quality of distribution is the sum of quality of beam section of the hull itself and the entrained water;

(4) Shear equivalent area of the beam is the average of areas on both ends of the beam section.

Generally, the longitudinal components throughout the length of the hull include bottom plate, deck, keelson, keelson, deck girder, the hatch coaming and side girder. The horizontal components include bottom plate, deck, floor board, deck beams, strong beam, hatch coaming, frame and strong frame. The transverse component is used to ensure the transverse stiffness of the hull.

Because most of the components are profiles, such as $\mathrm{T}$ steel and Angle steel, so its quality, the moment of inertia, cross-sectional area and section moment of inertia can be expressed by formula containing design variables. But the outside plates of irregular shape of boat, it can be obtained by the method of fitting expressions. The values of sectional moment of inertia of 20 sections respectively fill in the attribute of ADAMS.

In the solution of section parameters of beam element, the method of determining the moment of inertia and its orientation is studied [14]. X.Dong calculated the moment of inertia of car [15]. M.H.Li discussed the method for solving the moment of inertia of the cross section of the beam [16]. In this paper, taking a $54 \mathrm{~m}$ 
motor cargo ship as an example, the moment of inertia of each beam element is processed. Results are shown in table 1 3:

Table 1. Sectional moment of inertia of $\mathrm{z}$ axis.

\begin{tabular}{|c|c|c|c|}
\hline Num & $\mathbf{I z}(\mathbf{m m} 4)$ & Num & $\mathbf{I z}(\mathbf{m m 4})$ \\
\hline 1 & 22454743151558.6 & 11 & 2997200615982.70 \\
\hline 2 & 2969455466352.32 & 12 & 2997200615982.70 \\
\hline 3 & 3604974664103.87 & 13 & 2997200615982.70 \\
\hline 4 & 3223314900104.67 & 14 & 2997200615982.70 \\
\hline 5 & 3214244418809.15 & 15 & 2997200615982.70 \\
\hline 6 & 2997200615982.70 & 16 & 2997200615982.70 \\
\hline 7 & 2997200615982.70 & 17 & 4608184785593.97 \\
\hline 8 & 2997200615982.70 & 18 & 4340550638466.67 \\
\hline 9 & 2997200615982.70 & 19 & 1836116886963.17 \\
\hline 10 & 2997200615982.70 & 20 & 537280338616.40 \\
\hline
\end{tabular}

Table 2. Sectional moment of inertia of $\mathrm{x}$ axis.

\begin{tabular}{|c|c|c|c|}
\hline Num & Ix(mm4) & Num & Ix(mm4) \\
\hline 1 & 422242667234.485 & 11 & 503839201896.855 \\
\hline 2 & 638843182766.61 & 12 & 503839201896.855 \\
\hline 3 & 913628593998.18 & 13 & 503839201896.855 \\
\hline 4 & 875216217617.815 & 14 & 503839201896.855 \\
\hline 5 & 746077934134.25 & 15 & 503839201896.855 \\
\hline 6 & 503839201896.855 & 16 & 503839201896.855 \\
\hline 7 & 503839201896.855 & 17 & 882628554523.155 \\
\hline 8 & 503839201896.855 & 18 & 1003557535370.02 \\
\hline 9 & 503839201896.855 & 19 & 514515258500.11 \\
\hline 10 & 503839201896.855 & 20 & 136034778370.385 \\
\hline
\end{tabular}

Table 3. Sectional moment of inertia of y axis.

\begin{tabular}{|c|c|c|c|}
\hline Num & Iy(mm4) & Num & Iy(mm4) \\
\hline 1 & 2232500484325.1 & 11 & 2493361414085.7 \\
\hline 2 & 2330614418775.88 & 12 & 2493361414085.7 \\
\hline 3 & 2691348205294.77 & 13 & 2493361414085.7 \\
\hline 4 & 2348098682486.42 & 14 & 2493361414085.7 \\
\hline 5 & 2468166484674.42 & 15 & 2493361414085.7 \\
\hline 6 & 2493361414085.7 & 16 & 2493361414085.7 \\
\hline 7 & 2493361414085.7 & 17 & 3725563864677.87 \\
\hline 8 & 2493361414085.7 & 18 & 2229275264407.74 \\
\hline 9 & 2493361414085.7 & 19 & 1321601628467.21 \\
\hline 10 & 2493361414085.7 & 20 & 401245560250.7 \\
\hline
\end{tabular}

Multi-rigid-body piecewise model is established in ADAMS, which is connected by beam elements. The beam model of the hull as shown in Fig. 1.

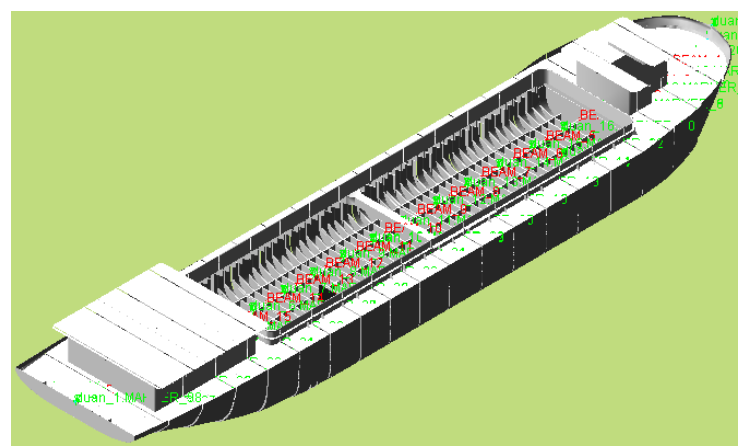

Figure 1. The model of Section beam element in ADAMS

Results of natural frequency of the hull beam model are obtained by running the program. The error of the results and the actual natural frequency are shown in Table 4.

Table 4. Sectional moment of inertia of y axis.

\begin{tabular}{|c|c|c|}
\hline $\begin{array}{c}\text { Actual natural } \\
\text { frequence }(\mathbf{H z})\end{array}$ & $\begin{array}{c}\text { Beam model } \\
\text { results }(\mathbf{H z})\end{array}$ & error\% \\
\hline 0.9953 & 1.125 & 13.0312 \\
\hline 1.3108 & 1.5176 & 15.7766 \\
\hline 2.1445 & 2.634 & 22.8258 \\
\hline 3.1305 & 3.8821 & 24.0089 \\
\hline 3.3807 & 4.3479 & 28.6095 \\
\hline 3.5282 & 4.6523 & 31.8604 \\
\hline 3.7679 & 4.9875 & 32.3682 \\
\hline 4.3084 & 5.7787 & 34.1264 \\
\hline 4.8068 & 6.4879 & 34.9734 \\
\hline 5.559 & 7.5946 & 36.6181 \\
\hline
\end{tabular}

As can be seen from the results, the error of hull simplified by this way is great.

\section{The processing of Lateral stiffness}

The simplified model of the hull beam by the former way ignores the effect of the transverse components to hull stiffness. When calculate the section, transverse components such as transverse beam and ribs in the two sections of the beam are often not cut, after division of beam element. The results calculated show that the stiffness of section inertia moment will be much smaller than the actual stiffness of sectional inertia moment. And even if either cross sections or one of them is on the transverse components, the calculated results and the actual situation still differs a lot. The stiffness of the frame structure with vertical and horizontal cross is different from that of the solid structure.

So the method of calculation of section moment of inertia of $y$ axis should be different from the $x$ and $z$ axis.

The method of calculation of the $\mathrm{x}$ and $\mathrm{z}$ axis remains the same, another method is chosen for y axis [17].

For any section of hull, sectional modulus can be expressed as:

$$
\begin{gathered}
W=\frac{Z}{G} \\
I_{y}=W \times y_{\max }=\frac{Z \times y_{\max }}{G} \\
Z=\sum_{i=1}^{n}\left[T_{Y i}\left(H_{U i}-H_{D i}\right)^{3} / 12\right. \\
\left.+T_{Y i}\left(H_{U i}-H_{D i}\right)\left(\left(H_{U i}+H_{D i}\right) / 2-G\right)^{2}\right] N_{Y i} \\
+\sum_{i=1}^{m} T_{X i} L_{X i}\left(X_{i}-G\right)^{2} N_{X i} \\
G=\frac{\sum_{i=1}^{m} T_{X i} L_{X i} X_{i} N_{X i}}{\sum_{i=1}^{m} T_{X i} L_{X i} N_{X i}+\sum_{i=1}^{n} T_{Y i}\left(H_{U i}-H_{D i}\right) N_{Y i}} \\
\sum_{i=1}^{n} T_{Y i}\left(H_{U i}-H_{D i}\right)\left(H_{U i}+H_{D i}\right) / 2 N_{Y i} \\
\sum_{i=1}^{m} T_{X i} L_{X i} N_{X i}+\sum_{i=1}^{n} T_{Y i}\left(H_{U i}-H_{D i}\right) N_{Y i}
\end{gathered}
$$

in the formula, 
$G$ - the height from the neutral axis to the base plate when cross section is in linear elastic range, $\mathrm{m}$;

$T_{X i}$ - The thickness of the class I components in the horizontal direction, $\mathrm{mm}$;

$L_{X i}$ - the length of the components of I class in the horizontal direction, $\mathrm{m}$;

$X_{i}$ - the height from the components of I class to the base plate, $\mathrm{m}$;

$N_{X i}$ - the number of the components of I class in the horizontal direction;

$T_{Y i}$ - the thickness of the components of I class in the vertical direction, $\mathrm{mm}$;

$H_{U i}, H_{D i}$ - the height from both ends of the components of I class to the base plate, $\mathrm{m}$;

$N_{Y i}$ - the number of the components of I class in the vertical direction;

$\mathrm{m}$ - the number kinds of horizontal components;

$\mathrm{n}$ - the number kinds of vertical components;

In order to achieve the purpose of high efficiency and simple, the program is prepared to calculate:

$>>$ syms tx 1 tx 2 tx 3 tx 4 tx 5 tx 6 lx 1 lx 2 lx 3 lx 4 lx 5 lx6 $\mathrm{x} 1 \mathrm{x} 2 \mathrm{x} 3 \mathrm{x} 4 \mathrm{x} 5 \mathrm{x} 6 \mathrm{nx} 1 \mathrm{nx} 2 \mathrm{nx} 3 \mathrm{nx} 4 \mathrm{nx} 5 \mathrm{nx} 6$ ty1 ty2 ty 3 ty4 ny1 ny2 ny3 ny4 hu1 hu2 hu3 hu4 hd1 hd2 hd3 hd4;

$>>\mathrm{G}=(\mathrm{tx} 1 * 1 \mathrm{x} 1 * \mathrm{x} 1 * \mathrm{nx} 1+\mathrm{tx} 2 * 1 \times 2 * \times 2 * \mathrm{n} \times 2+\mathrm{tx} 3 * 1 \times 3 * \mathrm{x} 3$ $*_{\mathrm{nx}} 3+\mathrm{tx} 4 * 1 \mathrm{x} 4 * \mathrm{x} 4 * \mathrm{nx} 4+\mathrm{tx} 5 * 1 \times 5 * \mathrm{x} 5 * \mathrm{nx} 5+\mathrm{tx} 6 * 1 \mathrm{x} 6 * \mathrm{x} 6 * \mathrm{nx}$ $6+$ ty $1 *($ hu $1-h d 1) *($ hu $1+$ hd 1$) / 2 /$ ny $1+$ ty $2 *($ hu $2-$

hd2)*(hu2+hd 2$) / 2 /$ ny $2+$ ty $3 *$ hu3 -

hd3)*(hu3+hd3)/2/ny3+ty $4 *$ (hu4-

hd4)*(hu4+hd4)/2/ny4)/(tx $1 * 1 \times 1 * n x 1+$ tx $2 * 1 \times 2 * n \times 2+t \times 3 *$ $1 \times 3 *$ nx $3+$ tx $4 * 1 \times 4 *$ nx $4+$ tx $5 * 1 \times 5 *$ nx $5+$ tx $6 * 1 \times 6 *$ nx $6+$ ty $1 *($ hu1-hd1)*ny1+ty $2 *($ hu2-hd2)*ny $2+$ ty $3 *($ hu3 -

hd3)*ny3+ty4*(hu4-hd4)*ny4);

$>>\mathrm{Z}=\mathrm{tx} 1 * 1 \mathrm{x} 1 *(\mathrm{x} 1-\mathrm{G}) \wedge 2 * \mathrm{nx} 1+\mathrm{tx} 2 * 1 \times 2 *(\mathrm{x} 2-$

$\mathrm{G})^{\wedge} 2 * \mathrm{n} \times 2+\mathrm{tx} 3 * 1 \times 3 *(\mathrm{x} 3-\mathrm{G})^{\wedge} 2 * \mathrm{n} \times 3+\mathrm{tx} 4 * 1 \times 4 *(\mathrm{x} 4-$

$\mathrm{G})^{\wedge} 2 * \mathrm{nx} 4+\mathrm{tx} 5 * 1 \times 5 *(\mathrm{x} 5-\mathrm{G})^{\wedge} 2 * \mathrm{nx} 5+\mathrm{tx} 6 * 1 \times 6 *(\mathrm{x} 6-$

$\mathrm{G})^{\wedge} 2 * \mathrm{nx} 6+\left(\right.$ ty $1 *(\text { hu } 1-\mathrm{hd} 1)^{\wedge} 3 / 12+$ ty $1 *$ (hu $1-$

hd1 $)^{*}(($ hu $1+$ hd 1$\left.) / 2-G) \wedge 2\right)^{*}$ ny $1+($ ty $2 *($ hu $2-$

hd2 $)^{\wedge} 3 / 12+$ ty $2 *($ hu $2-$ hd 2$) *(($ hu $2+$ hd 2$) / 2-$

$\left.\mathrm{G})^{\wedge} 2\right)^{*}$ ny $2+\left(\right.$ ty $3 *(\text { hu } 3-\text { hd } 3)^{\wedge} 3 / 12+$ ty $3 *($ hu $3-$

hd3 $)^{*}(($ hu3 $3+$ hd3 $\left.) / 2-G) \wedge 2\right) *$ ny3+(ty4*(hu4-

hd4 $)^{\wedge} 3 / 12+$ ty $4 *($ hu $\left.4-h d 4) *((\text { hu } 4+\text { hd } 4) / 2-G)^{\wedge} 2\right)^{*}$ ny 4 ;

$>\mathrm{W}=\mathrm{Z} / \mathrm{G}$;

Set the section moment of inertia of $y$ axis after integration into the attributes of the software ADAMS, calculating along with the data of the $\mathrm{x}, \mathrm{z}$ axis.

Results as shown in table 5:

Table 5. beam model results of sectional moment of inertia of y axis after changed and the error of the actual natural frequency

\begin{tabular}{|c|c|c|}
\hline $\begin{array}{c}\text { Actual natural } \\
\text { frequence }(\mathbf{H z})\end{array}$ & $\begin{array}{c}\text { Beam model } \\
\text { results }(\mathbf{H z})\end{array}$ & error\% \\
\hline 0.9953 & 1.0252 & 3.0041 \\
\hline 1.3108 & 1.3626 & 3.9518 \\
\hline 2.1445 & 2.2313 & 4.0476 \\
\hline 3.1305 & 3.2576 & 4.0601 \\
\hline 3.3807 & 3.5724 & 5.6704 \\
\hline 3.5282 & 3.7588 & 6.5359 \\
\hline 3.7679 & 4.0731 & 8.1 \\
\hline
\end{tabular}

\begin{tabular}{|c|c|c|}
\hline 4.3084 & 4.8248 & 11.9859 \\
\hline 4.8068 & 5.4389 & 13.1501 \\
\hline 5.559 & 6.6472 & 19.5755 \\
\hline
\end{tabular}

From the data table can be seen, after changing the $\mathrm{Y}$ axis cross section moment of inertia calculation method, the accuracy of the results increased about 10 percentage points.

\section{The processing of other special parts}

When faced with ship with large opening, the ship is extremely prone to torsional deformation [18]. When the torsion occurs, the accuracy of simplified stiffness of $\mathrm{Z}$ axis is very important. It needs to make corresponding changes to calculate sectional moment of inertia of the $\mathrm{z}$ axis.

For analysis of hull torsion, the hull must be looked as thin-walled beam, deck and the outer plate as thin wall of hollow beam [19]. When the hull of the deck with a long opening has torsional deformation, section torsional moment of inertia:

$$
I_{z}=\frac{1}{3}\left(B t_{b}^{3}+2 D t_{s}^{2}+2 C t_{d}^{3}\right)
$$

in formula,

$$
\begin{aligned}
& \mathrm{B} \text { - The actual width of the hull, } \mathrm{m} ; \\
& \mathrm{D}-\text { The actual depth of the hull, } \mathrm{m} ; \\
& \mathrm{C} \text { - The width of the deck plate, } \mathrm{m} ; \\
& t_{b} \text { - The thickness of bottom plate, } \mathrm{mm} \text {; } \\
& t_{s} \text {-The thickness of side plate, } \mathrm{mm} ; \\
& t_{d} \text { - The thickness of deck plate, } \mathrm{mm} \text {. }
\end{aligned}
$$

This paper studied $54 \mathrm{~m}$ mobile carrier with the large opening, so the processing opening of section moment of inertia is considered.

After the sectional moment of inertia reprocessed and running in ADAMS, the results are as shown in table 6:

Table 6. beam model results of sectional moment of inertia of $z$ axis after changed and the error of the actual natural frequency

\begin{tabular}{|c|c|c|}
\hline $\begin{array}{c}\text { Actual natural } \\
\text { frequence }(\mathbf{H z})\end{array}$ & $\begin{array}{c}\text { Beam model } \\
\text { results }(\mathbf{H z})\end{array}$ & error\% \\
\hline 0.9953 & 1.0157 & 2.0496 \\
\hline 1.3108 & 1.3514 & 3.0973 \\
\hline 2.1445 & 2.2171 & 3.3854 \\
\hline 3.1305 & 3.2393 & 3.4755 \\
\hline 3.3807 & 3.4565 & 2.2421 \\
\hline 3.5282 & 3.6681 & 3.9652 \\
\hline $\begin{array}{c}\text { Actual natural } \\
\text { frequence }(\mathbf{H z})\end{array}$ & $\begin{array}{c}\text { Beam model } \\
\text { results }(\mathbf{H z})\end{array}$ & error\% \\
\hline 3.7679 & 3.9753 & 5.5044 \\
\hline 4.3084 & 4.6149 & 7.114 \\
\hline 4.8068 & 5.2466 & 9.1495 \\
\hline 5.559 & 6.2308 & 12.0849 \\
\hline
\end{tabular}

It is can be seen from the results that after the processing changed, the accuracy of the sectional moment of inertia improves again. All the results of beam model and the error with actual natural frequency are all 
reduced by $1 \%$ or so. And they are more close to actual results.

\section{Conclusions}

The processing of equivalent stiffness is a very important research part in vibration analysis. The accuracy of the equivalent stiffness directly affects the results of the entire study whether is correct and whether the conclusion of the research makes sense.

In this paper, the processing method of the equivalent stiffness of the ship hull girder is studied. Using ADAMS software for auxiliary calculation obtained more accurate method than the commonly used method to deal with hull girder segment element. The equivalent stiffness is more close to the actual situation, thus improving the accuracy of the calculation of natural frequency in the study of vibration characteristics. Having been used in ADAMS, the modelling method turns out to be more accurate processing method than common hull girder section unit method.

The method is applicable for any hull which can be simplified to beam model. In this paper, the method of processing thin-walled structures with transverse and longitudinal structure can also provide a kind of support for the research of other subjects.

\section{Acknowledgements}

This work got some good people's helps. Thanks for my teacher, all my brothers and my sisters. All those who contributed directly or indirectly are thanked.

\section{References}

1. Z.Q.Li, Study on Ship Hull Vibration Model Building(Dalian University of Technology,2006)(In Chinese)

2. Y.M.Yin, Study on Modeling Method of Ship Vibration(Dalian University of Technology,2007) (In Chinese)

3. X.Z.Wang, The study of hull vibration characteristics (Dalian University of Technology,2006)(In Chinese)

4. H.Cheng,Study on the vibration of the three body ship attached to the water quality( Wuhan University of Technology, 2011) (In Chinese)

5. X.Wei,Study on Experiment and Numerical Simulation of Added Water Mass for Trimaran
Vibration(Wuhan University of Technology,2012) (In Chinese)

6. Z.W.Chen, Y.L.Dong, Multi body dynamics simulation based on MSC Adams(China Water Conservancy and Hydropower Press, BeiJing,2012)

7. W.M.Ji, T.Fang, S.Q.Chen, Mechanical vibration (Science Press, Beijing, 1985) (In Chinese)

8. C.Luo,C.P.Zou,Z.G.Li, Analysis of ship stern shaft bracket system modeling and vibration characteristics,Chin J Ship Res (2),55-61(2014) (In Chinese)

9. X.Peng,D.P.Luo, Modeling of ship structure and prediction of underwater vibration and radiation noise, Noise and Vibration Control 23(06),9-12(2003) (In Chinese)

10. R.W.Che,N.L.Lu, A new method for dynamic modeling of multibody system,Turbine technology, 45(6),364-365(2003) (In Chinese)

11. Y.M.Yin,D.Y.Zhao, Research on finite element modeling method for overall vibration of Ship Superstructure, Shipbuilding of China50(03),4956(2009) (In Chinese)

12. X.T.Rui, W.H.Sui, Y.Z.Shao, The field transfer matrix and rigid multi-body dynamics in the application, Journal of Astronautics 4,82-87(1993) (In Chinese)

13. X.D.Jin, J.Xia, Ship vibration (Shanghai Jiao Tong University press, Shanghai, 2011) (In Chinese)

14. J.Du, Research on Modeling and Simulation of simplified car body based on a passenger car(Jilin University,Jilin,2012)(In Chinese)

15. X.Dong, Calculation and FEM analysis of simplified model based on derivation of rigidity matrix(Jilin University,Jilin,2004)(In Chinese)

16. M.H.Li,Discussion on the method for solving the moment of inertia of the cross section of the beam,Gansu Science and Technology23(2),145146(2007) (In Chinese)

17. Z.M.Ma,Analysis of ultimate strength of ship hull girder(Wuhan University of Technology, Wuhan, 2003) (In Chinese)

18. F.Lin, Analysis of the structural characteristics of 50000 tons large open container / bulk carrier, Journal of Marine Science and Application 1 ,3639(1996) (In Chinese)

19. Y.H.Xie, J.G.Wu, J.L.Li, Design of ship structure (Publishing House of Shanghai Jiao Tong University, Shanghai,2011) (In Chinese) 